\title{
1 Over-expression of non-SMC condensin I complex subunit G in human endometrial cancer. \\ Shahan Mamoor, $\mathrm{MS}^{1}$ \\ ${ }^{1}$ shahanmamoor@gmail.com \\ $4 \quad$ East Islip, NY USA \\ Gynecologic cancers including cancers of the endometrium are a clinical problem ${ }^{1-4}$. We mined published microarray data ${ }^{5,6}$ to discover genes associated with endometrial cancers by comparing transcriptomes of the normal endometrium and endometrial tumors from humans. We identified non-SMC condensin I complex subunit G, encoded by NCAPG, as among the most differentially expressed genes, transcriptome-wide, in cancers of the endometrium. NCAPG was expressed at significantly higher levels in endometrial tumor tissues as compared to the endometrium. Importantly, in human endometrial cancer, primary tumor expression of NCAPG was correlated with recurrence-free survival in white patients with low mutational burden. NCAPG may be a molecule of interest in understanding the etiology or progression of human endometrial cancer.
}

Keywords: endometrial cancer, gynecologic cancers, endometrium, NCAPG, non-SMC condensin I complex subunit G, systems biology of endometrial cancer, targeted therapeutics in endometrial cancer. 
Endometrial cancer is the most common gynecologic cancer in the developed world ${ }^{1}$. Over the last three decades, the incidence of endometrial cancer has increased $21 \%{ }^{4}$ and the death rate has increased $100 \%{ }^{3}$. We harnessed the power of independently published microarray datasets $^{5,6}$ to determine in an unbiased fashion and at the systems-level genes most differentially expressed in endometrial tumors. We report here the differential and increased expression of the non-SMC condensin I complex subunit G (NCAPG) in human endometrial cancer.

\section{Methods}

We utilized datasets GSE63678 5 and GSE17025 ${ }^{6}$ for this global differential gene expression analysis of human endometrial cancer in conjunction with GEO2R. GSE63678 was generated using Affymetrix Human Genome U133A 2.0 Array technology with $n=5$ control endometrial tissues (including $n=4$ uterine myomas and $n=1$ benign cyst) and $n=7$ endometrial cancers (including $n=2$ endometrial adenocarcinomas, $n=3$ mixed endometrioid adenocarcinomas, and $n=2$ adenocarcinomas with squamous differentiation); analysis was performed using platform GPL571. GSE17025 was generated using Affymetrix Human Genome U133 Plus 2.0 Array technology with $n=5$ normal endometrial tissues (inactive endometrium) and $n=91$ endometrial cancers; analysis was performed using platform GPL570. The Benjamini and Hochberg method of $p$-value adjustment was used for ranking of differential expression but raw $p$-values were used to assess statistical significance of global differential expression.

Log-transformation of data was auto-detected, and the NCBI generated category of platform annotation was used. A statistical test was performed to evaluate whether NCAPG gene expression was significantly different between control endometrial tissue and endometrial tumor tissue in humans using a two-tailed t-test. For Kaplan-Meier survival analysis, we used the Kaplan-Meier plotter tool ${ }^{7}$ for correlation of NCAPG mRNA expression levels with recurrence-free survival in $n=543$ endometrial cancer patients.

\section{$\underline{\text { Results }}$}

We harnessed the power of blind comparative transcriptome analysis using published microarray data ${ }^{5,6}$ to discover in an unbiased fashion genes associated with endometrial cancer in humans.

\section{NCAPG is differentially expressed in endometrial cancer.}

We identified non-SMC condensin I complex subunit G, encoded by NCAPG, as among the genes most differentially expressed in cancers of the endometrium when compared to benign endometrial tissues (Chart 1). When sorting each of the genes expressed in endometrial tumor tissue based on significance of change in expression as compared to benign endometrial tissue, NCAPG ranked 76 out of 22273 transcripts, equating to $99.7 \%$ differential expression (Chart 1). Differential expression of NCAPG in human endometrial cancers was statistically significant (Chart $1 ; p=7.01 \mathrm{E}-05$ ). 
We queried a second microarray data to validate differential expression of NCAPG in endometrial cancer. Again, we observed differential expression of NCAPG when comparing endometrial tumor tissue to benign endometrial tissue (Chart 2). When sorting each of the genes expressed in endometrial tumor tissue based on significance of change in expression as compared to benign endometrial tissue, NCAPG ranked 40 out of 54675 transcripts, equating to $99.9 \%$ differential expression (Chart 2). Differential expression of NCAPG in human endometrial cancers was statistically significant (Chart $2 ; p=3.76 \mathrm{E}-09)$.

\section{NCAPG is expressed at significantly higher levels in endometrial cancers as compared to benign endometrial tissue.}

We obtained exact mRNA expression levels for NCAPG in endometrial tumor tissues and from benign endometrial tissue to evaluate direction and statistical significance of change in expression of NCAPG in human endometrial cancer. NCAPG was expressed at higher levels in endometrial tissue as compared to normal endometrial tissue, and this difference was statistically significant (Figure 1; $p<0.0001$ ). We calculated a mean fold change of 1.38 in NCAPG mRNA levels in human endometrial cancer, as NCAPG was expressed at $5.36 \pm 0.36$ arbitrary units (A.U.) in control endometrial tissue but at $7.39 \pm 0.76$ A.U. in endometrial tumor tissue.

\section{NCAPG expression is correlated with patient survival outcomes in endometrial cancer.}

We performed Kaplan-Meier survival analysis to evaluate correlation between NCAPG primary tumor expression and survival outcomes in 543 patients with endometrial cancer. We observed a correlation between primary tumor expression of NCAPG and recurrence-free survival in patients with endometrial cancer, in white patients with low mutational burden (Figure 2). NCAPG primary tumor mRNA levels were a negative prognostic indicator in white endometrial cancer patients with low mutational burden. White patients with low mutational burden whose primary tumors expressed low levels of NCAPG possessed median RFS of 78.4 months, while white patients with low mutational burden whose tumors expressed high levels of NCAPG possessed median RFS of 32.97 months. This difference in RFS based on NCAPG tumor expression in white patients with endometrial cancer with low mutational burden approached statistical significance (Figure 2, Chart 3; logrank p-value: 0.00029; hazard ratio: 3.94 (1.77-8.75)). NCAPG primary endometrial tumor expression was not correlated with recurrence-free survival in white patients with high mutational burden (Figure 2, Chart 3; logrank p-value: 0.84; hazard ratio: $1.08(0.49-2.38))$, nor in black patients with high ((Figure 2, Chart 3; logrank $p$-value: 0.98; hazard ratio: $1.01(0.34-3.04)$ ) or low mutational burden (Figure 2, Chart 3 ; logrank $p$-value: 0.87 ; hazard ratio: $0.87(0.17-4.35))$.

Thus, by mining published microarray data ${ }^{5,6}$ in an unbiased and systematic fashion, we identified non-SMC condensin I complex subunit G, encoded by NCAPG, as among the genes whose expression was most different, transcriptome-wide, in the endometrial tumor tissue of 


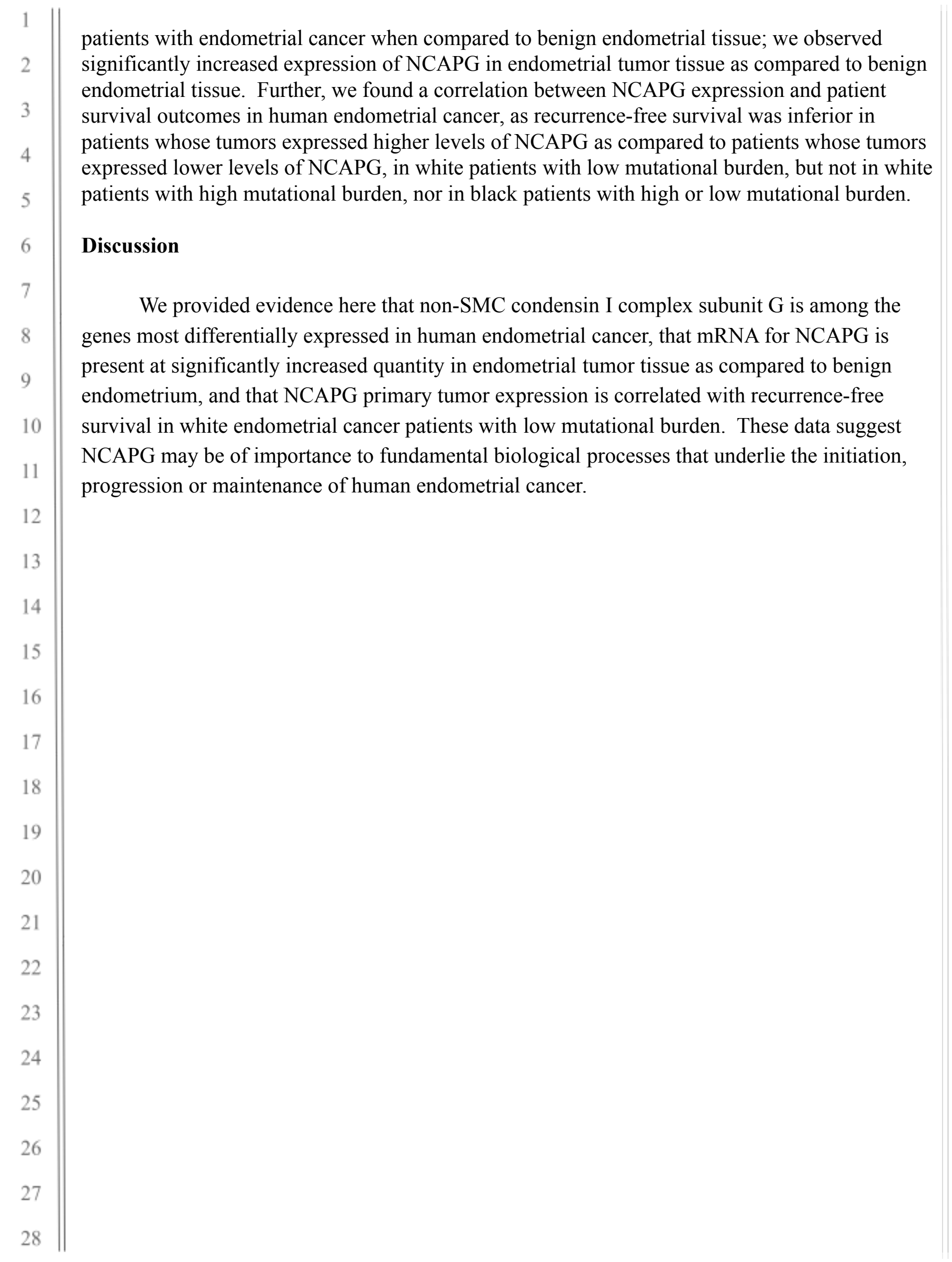




\section{References}

1. Amant, F., Moerman, P., Neven, P., Timmerman, D., Van Limbergen, E. and Vergote, I., 2005. Endometrial cancer. The Lancet, 366(9484), pp.491-505.

2. Sorosky, J.I., 2008. Endometrial cancer. Obstetrics \& Gynecology, 111(2), pp.436-447.

3. Morice, P., Leary, A., Creutzberg, C., Abu-Rustum, N. and Darai, E., 2016. Endometrial cancer. The Lancet, 387(10023), pp.1094-1108.

4. Sorosky, J.I., 2012. Endometrial cancer. Obstetrics \& Gynecology, 120 (2 Part 1), pp.383-397.

5. Pappa, K.I., Polyzos, A., Jacob-Hirsch, J., Amariglio, N., Vlachos, G.D., Loutradis, D. and Anagnou, N.P., 2015. Profiling of discrete gynecological cancers reveals novel transcriptional modules and common features shared by other cancer types and embryonic stem cells. PLoS One, 10(11), p.e0142229.

6. Day, R.S., McDade, K.K., Chandran, U.R., Lisovich, A., Conrads, T.P., Hood, B.L., Kolli, V.K., Kirchner, D., Litzi, T. and Maxwell, G.L., 2011. Identifier mapping performance for integrating transcriptomics and proteomics experimental results. BMC bioinformatics, 12(1), p.213.

7. Nagy, A., Munkacsy, G. and Gyorffy, B., 2020. Pancancer survival analysis of cancer hallmark genes. bioRxiv. 


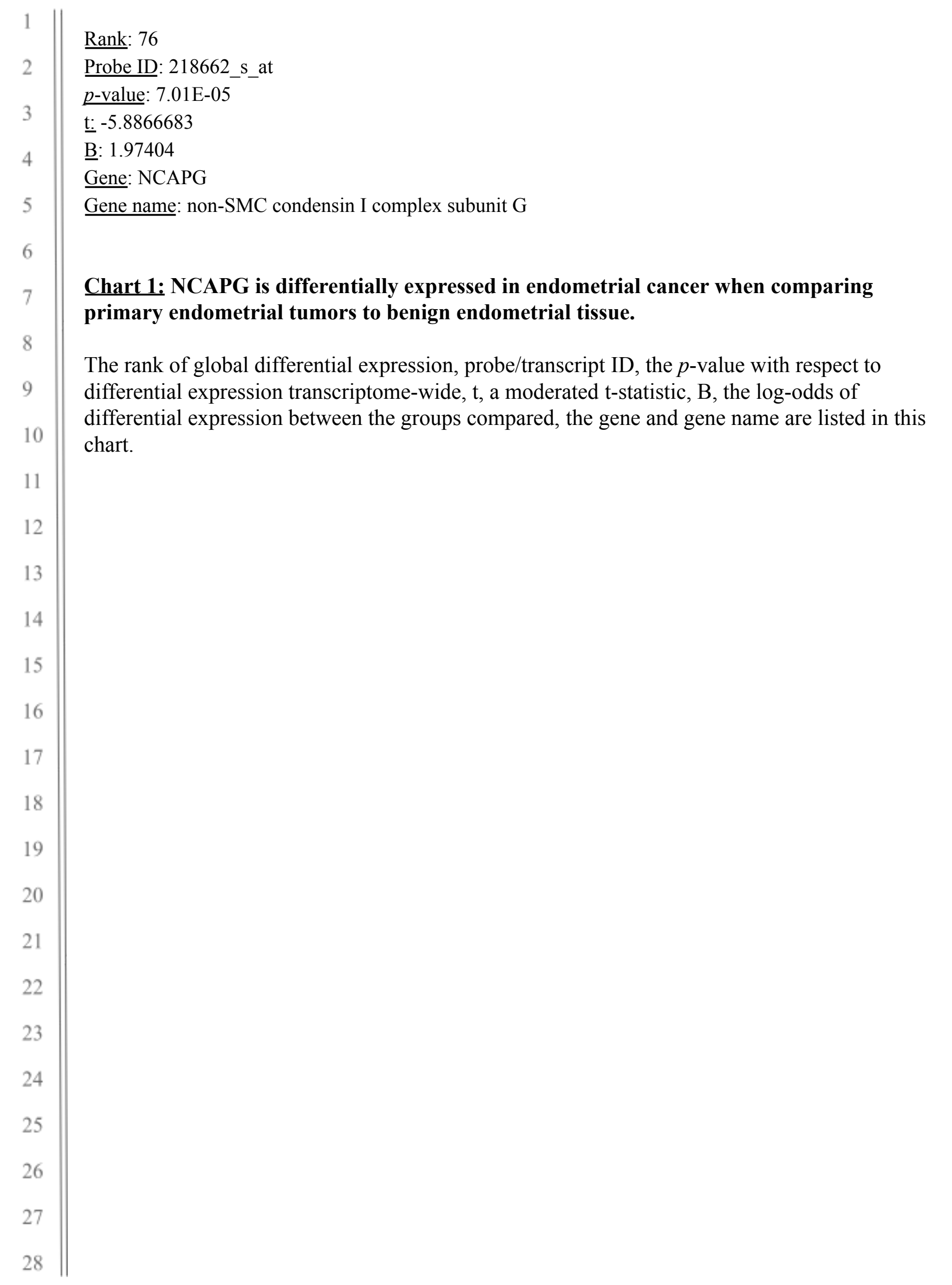




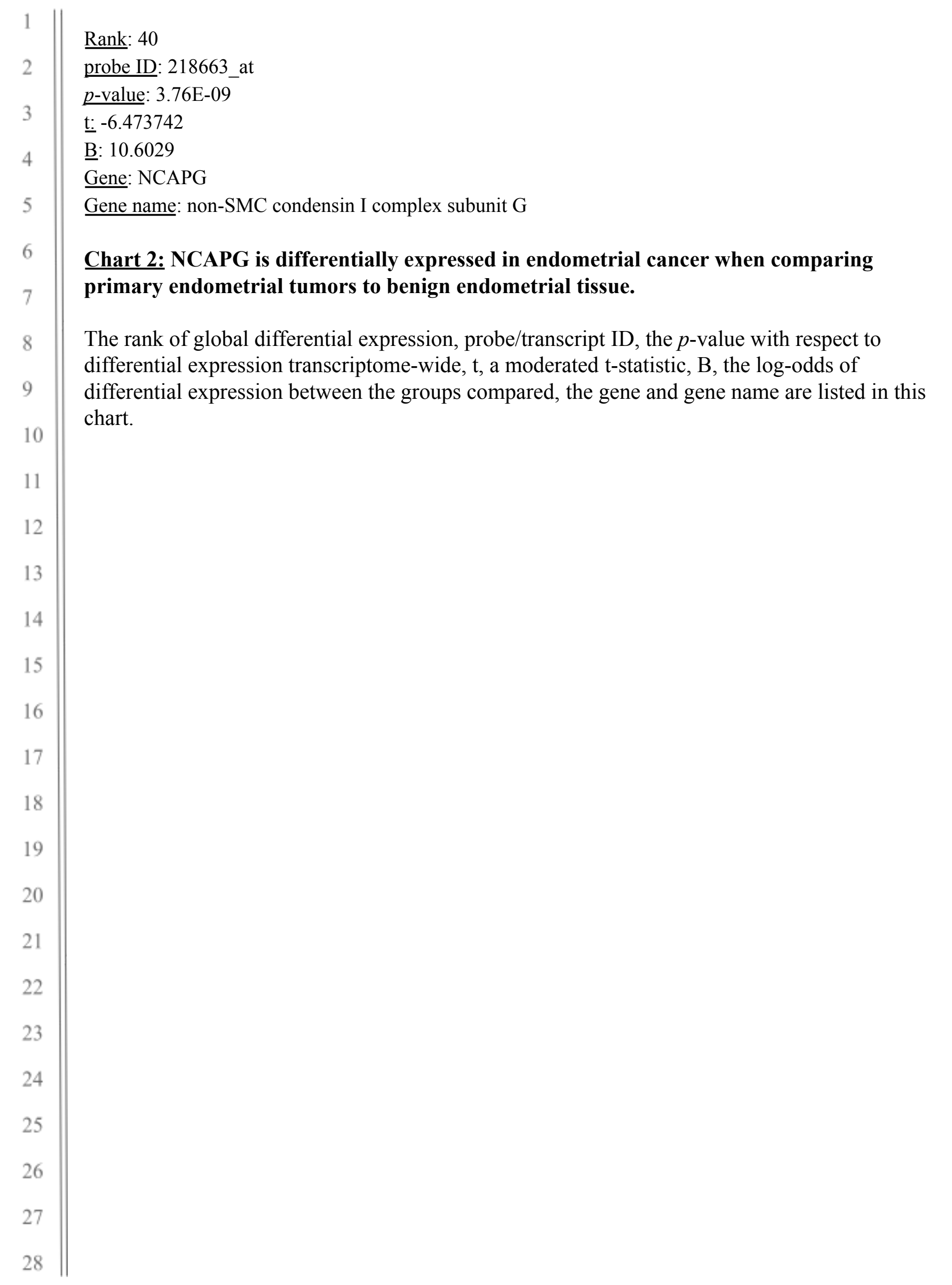




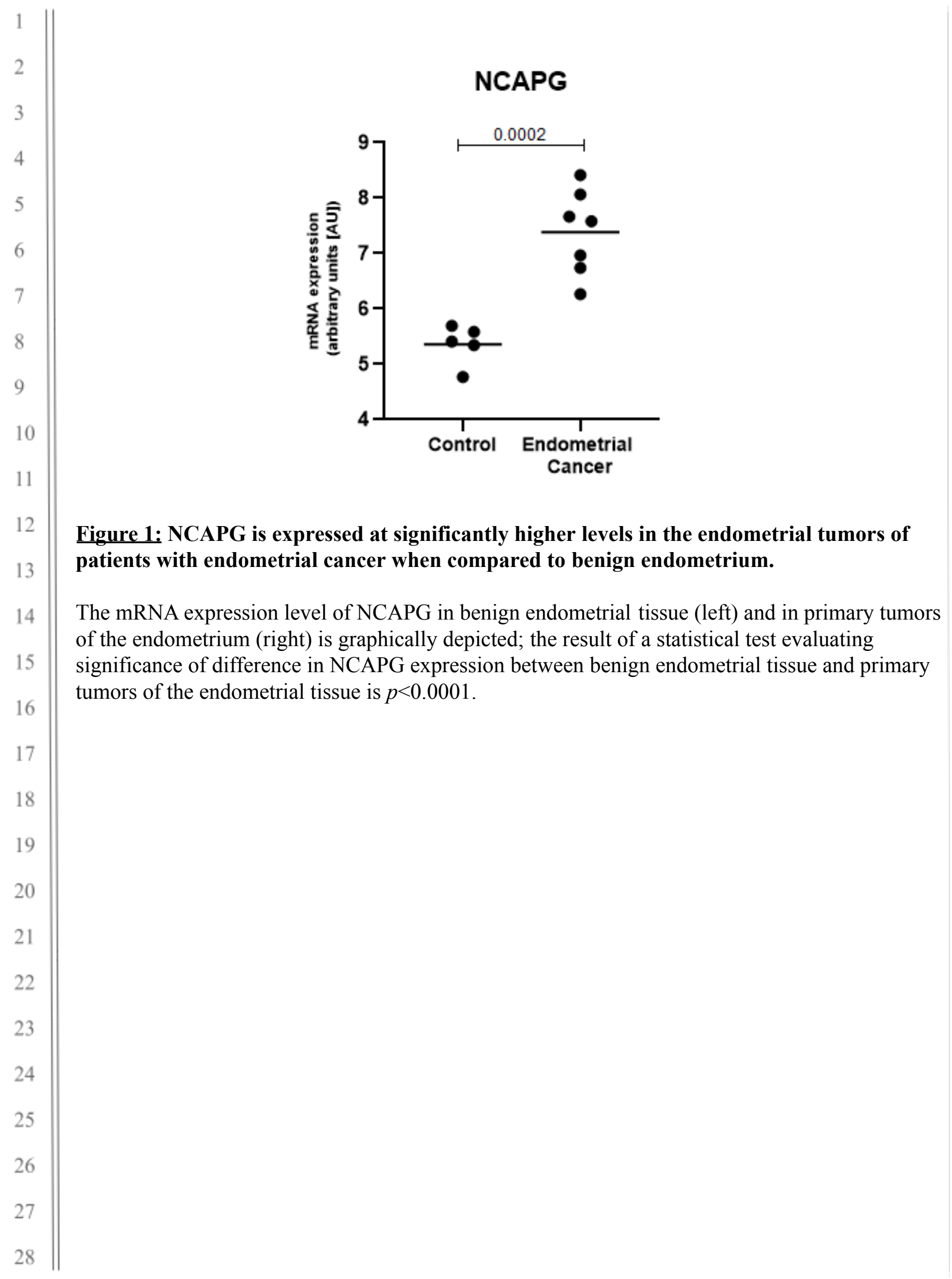



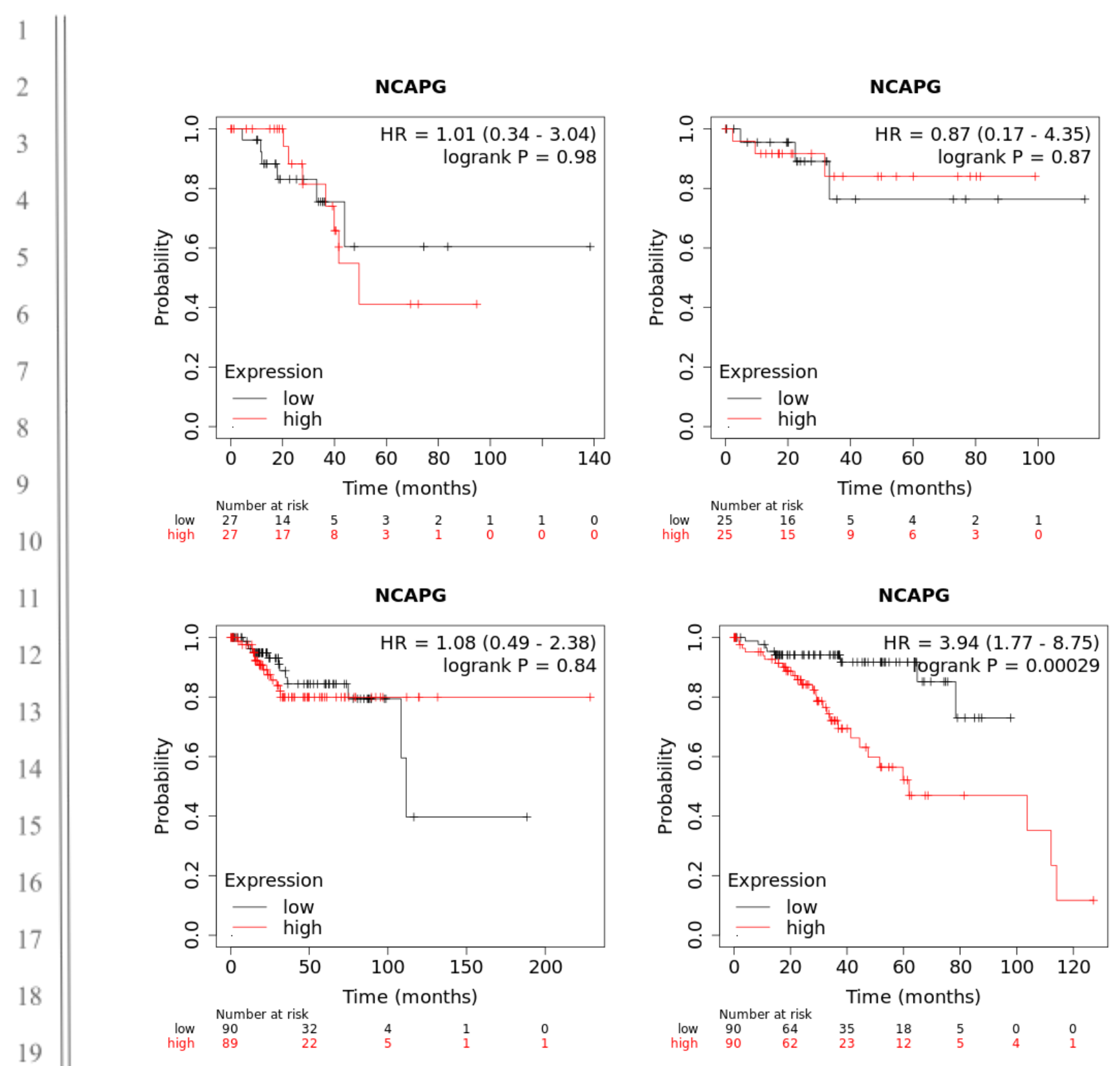

Figure 2: Correlation between NCAPG primary tumor expression and recurrence-free survival in endometrial cancer, in white patients with low mutational burden.

Depicted in this Kaplan-Meier plot is the probability of recurrence-free survival for $n=543$ total endometrial cancer patients stratified into two groups, based on low or high expression of NCAPG in patient primary tumors, in black patients with high mutational burden (top left), black patients with low mutational burden (top right), white patients with high mutational burden (bottom left), and white patients with low mutational burden (bottom right). The log rank $p$-value denoting statistical significance of difference in recurrence-free survival when comparing the two groups, as well as hazard ratio for this comparison is listed above. Listed below is the number of patients at risk (number of patients alive) per interval, after stratification based on NCAPG expression; in the first interval, number at risk is number of patients alive; in each subsequent interval, number at risk is the number at risk less those who have expired or are censored. 
Low NCAPG expression: 78.4 months

High NCAPG expression: 32.97 months

3

4

5

6

7
Chart 3: Median recurrence-free survival is superior in endometrial cancer patients with low primary tumor expression of NCAPG, in white patients with low mutational burden.

The median RFS (recurrence-free survival) of white endometrial cancer patients with low mutational burden, with low primary tumor expression of NCAPG and high primary tumor expression of NCAPG is listed in this chart. 